\title{
VB-Courant algebroids, E-Courant algebroids and generalized geometry *
}

\author{
Honglei Lang ${ }^{1}$, Yunhe Sheng ${ }^{2}$ and Aissa Wade ${ }^{3}$ \\ ${ }^{1}$ Max Planck Institute for Mathematics, \\ Bonn 53111, Germany \\ ${ }^{2}$ Department of Mathematics, Jilin University, \\ Changchun 130012, Jilin, China \\ ${ }^{3}$ Mathematics Department, Penn State University, \\ University Park, PA 16802, USA. \\ email: hllang@mpim-bonn.mpg.de, shengyh@jlu.edu.cn, wade@math.psu.edu
}

\begin{abstract}
In this paper, we first discuss the relation between VB-Courant algebroids and E-Courant algebroids and construct some examples of E-Courant algebroids. Then we introduce the notion of a generalized complex structure on an E-Courant algebroid, unifying the usual generalized complex structures on even-dimensional manifolds and generalized contact structures on odd-dimensional manifolds. Moreover, we study generalized complex structures on an omni-Lie algebroid in detail. In particular, we show that generalized complex structures on an omni-Lie algebra $\mathrm{gl}(V) \oplus V$ correspond to complex Lie algebra structures on $V$.
\end{abstract}

\section{Introduction}

The theory of Courant algebroids was first introduced by Liu, Weinstein and Xu [17] providing an extension of Drinfeld's double for Lie bialgebroids. Precisely, the double of a Lie bialgebroid is a special Courant algebroid [17, 20. Jacobi algebroids are natural extensions of Lie algebroids and Courant-Jacobi algebroids. They were considered by Grabowski and Marmo [7] and they can be viewed as generalizations of Courant algebroids. Both Courant algebroids and Courant-Jacobi algebroids have been extensively studied in the last decade since these are crucial geometric tools in Poisson geometry and mathematical physics. It is known that they both belong to a more general framework, namely that of E-Courant algebroids. Indeed, E-Courant algebroids were introduced by Chen, Liu and the second author in [5] as a differential geometric object encompassing Courant algebroids [17, Courant-Jacobi algebroids [7, omni-Lie algebroids [4, conformal Courant algebroids [2] and $A V$-Courant algebroids [14]. It turns out that E-Courant algebroids are related to more geometric structures such as VB-Courant algebroids [15].

The aim of this paper is two-fold. Firstly, we illuminate the relationship between VB-Courant algebroids and E-Courant algebroids. Secondly, we study generalized complex structures on E-

\footnotetext{
${ }^{0}$ Keyword: VB-Courant algebroid, E-Courant algebroid, omni-Lie algebroid, generalized complex structure, algebroid-Nijenhuis structure

${ }^{0} M S C$ : Primary 53D17. Secondary 18B40, 58H05.

* Research supported by NSFC (11471139) and NSF of Jilin Province (20170101050JC).
} 
Courant algebroids. Recall that a generalized almost complex structure on a manifold $M$ is an endomorphism $\mathcal{J}$ of the generalized tangent bundle $\mathbb{T} M:=T M \oplus T^{*} M$ which preserves the natural pairing on $\mathbb{T} M$ and such that $\mathcal{J}^{2}=-$ id. If, additionally, the $\sqrt{-1}$-eigenbundle of $\mathcal{J}$ in the complexification $\mathbb{T} M \otimes \mathbb{C}$ is involutive relative to the Dorfman (equivalently, the Courant) bracket, then $\mathcal{J}$ is said to be integrable, and $(M, \mathcal{J})$ is called a generalized complex manifold. See [3, 6, 8, 9, 23, for more details.

Given a vector bundle $E \stackrel{q}{\rightarrow} M$, we consider its gauge Lie algebroid $\mathfrak{D} E$, i.e. the gauge Lie algebroid of the frame bundle $\mathcal{F}(E)$. It is known that $\mathfrak{D} E$ is a transitive Lie algebroid over $M$ and the first jet bundle $\mathfrak{J} E$ is its $E$-dual bundle. In fact, ofl $(E)=\mathfrak{D} E \oplus \mathfrak{J} E$ is called an omniLie algebroid [4, which is a generalization of Weinstein's concept of an omni-Lie algebra [26]. In particular, the line bundle case where $E$ comes from a contact distribution brings us to the concept of a generalized contact bundle. To have a better grasp of the concept of a generalized contact bundle, we briefly review the line bundle approach to contact geometry. By definition, a contact structure on an odd-dimensional manifold $M$ is a maximal non-integrable hyperplane distribution $H \subset T M$. In a dual way, any hyperplane distribution $H$ on $M$ can be regarded as a nowhere vanishing 1-form $\theta: T M \rightarrow L$ (its structure form) with values in the line bundle $L=T M / H$, such that $H=\operatorname{ker} \theta$. Replacing the tangent algebroid with the Atiyah algebroid of a line bundle in the definition of a generalized complex manifold, we obtain the notion of a generalized contact bundle. In this paper, we extend the concept of a generalized contact bundle to the context of E-Courant algebroids.

The paper is organized as follows. Section 2 contains basic definitions used in the sequel. Section 3 highlights the importance and naturality of the notion of E-Courant algebroids. Explicitly, the fat Courant algebroid associated to a VB-Courant algebroid (see the definition of a VB-Courant algebroid below) is an E-Courant algebroid. We observe the following facts:

- Given a crossed module of Lie algebras $(\mathfrak{m}, \mathfrak{g})$, we get an $\mathfrak{m}$-Courant algebroid $\operatorname{Hom}(\mathfrak{g}, \mathfrak{m}) \oplus \mathfrak{g}$, which was given in [13] as a generalization of an omni-Lie algebra.

- The omni-Lie algebroid $\mathfrak{o l}(E)=\mathfrak{D} E \oplus \mathfrak{J} E$ is the linearization of the VB-Courant algebroid $T E^{*} \oplus T^{*} E^{*}$. This generalizes the fact that an omni-Lie algebra is the linearization of the standard Courant algebroid.

- For a Courant algebroid $\mathcal{C}, T \mathcal{C}$ is a VB-Courant algebroid. The associated fat Courant algebroid $\mathfrak{J C}$ is a $T^{*} M$-Courant algebroid. The fact that $\mathfrak{J C}$ is a $T^{*} M$-Courant algebroid was first obtained in [5, Theorem 2.13].

In Section 4, we introduce generalized complex structures on E-Courant algebroids and provide examples. In Sections 5, we describe generalized complex structures on omni-Lie algebroids. In Section 6, we show that generalized complex structures on the omni-Lie algebra or $(V)$ are in oneto-one correspondence with complex Lie algebra structures on $V$.

\section{Preliminaries}

Throughout the paper, $M$ is a smooth manifold, $\mathrm{d}$ is the usual differential operator on forms, and $E \longrightarrow M$ is a vector bundle. In this section, we recall the notions of E-Courant algebroids introduced in [5], omni-Lie algebroids introduced in 4, generalized complex structures introduced in [8, 9] and generalized contact structures introduced in [24]. 


\section{$2.1 \quad$ E-Courant algebroids and omni-Lie algebroids}

For a vector bundle $E \rightarrow M$, its gauge Lie algebroid $\mathfrak{D} E$ with the commutator bracket $[\cdot, \cdot]_{\mathfrak{D}}$ is just the gauge Lie algebroid of the frame bundle $\mathcal{F}(E)$, which is also called the covariant differential operator bundle of $E$ (see [18, Example 3.3.4]). The corresponding Atiyah sequence is as follows:

$$
0 \longrightarrow \operatorname{gl}(E) \stackrel{\text { i }}{\longrightarrow} \mathfrak{D} E \stackrel{\dot{j}}{\longrightarrow} T M \longrightarrow 0 .
$$

In [4, the authors proved that the jet bundle $\mathfrak{J} E$ may be considered as an $E$-dual bundle of $\mathfrak{D} E$ :

$$
\mathfrak{J} E \cong\left\{\nu \in \operatorname{Hom}(\mathfrak{D} E, E) \mid \nu(\Phi)=\Phi \circ \nu\left(\mathrm{id}_{E}\right), \quad \forall \Phi \in \operatorname{gl}(E)\right\} .
$$

Associated to the jet bundle $\mathfrak{J} E$, there is a jet sequence given by:

$$
0 \longrightarrow \operatorname{Hom}(T M, E) \stackrel{\mathbb{e}}{\longrightarrow} \mathfrak{J} E \stackrel{\mathrm{p}}{\longrightarrow} E \longrightarrow 0 .
$$

Define the operator $\mathbb{d}: \Gamma(E) \rightarrow \Gamma(\mathfrak{J} E)$ by:

$$
\mathbb{d} u(\mathfrak{d}):=\mathfrak{d}(u), \quad \forall u \in \Gamma(E), \mathfrak{d} \in \Gamma(\mathfrak{D} E) .
$$

An important formula which will be often used is

$$
\mathbb{d}(f u)=\mathrm{d} f \otimes u+f \mathbb{d} u, \quad \forall u \in \Gamma(E), f \in C^{\infty}(M) .
$$

In fact, there is an $E$-valued pairing between $\mathfrak{J} E$ and $\mathfrak{D} E$ by setting:

$$
\langle\mu, \mathfrak{d}\rangle_{E} \triangleq \mathfrak{d}(u), \quad \forall \mu \in(\mathfrak{J} E)_{m}, \mathfrak{d} \in(\mathfrak{D} E)_{m},
$$

where $u \in \Gamma(E)$ satisfies $\mu=[u]_{m}$. In particular, one has

$$
\begin{aligned}
\langle\mu, \Phi\rangle_{E} & =\Phi \circ \mathfrak{p}(\mu), \quad \forall \Phi \in \operatorname{gl}(E), \mu \in \mathfrak{J} E \\
\langle\mathfrak{y}, \mathfrak{d}\rangle_{E} & =\mathfrak{y} \circ \dot{\mathfrak{j}}(\mathfrak{d}), \quad \forall \mathfrak{y} \in \operatorname{Hom}(T M, E), \mathfrak{d} \in \mathfrak{D} E .
\end{aligned}
$$

For vector bundles $P, Q$ over $M$ and a bundle map $\rho: P \longrightarrow Q$, we denote the induced $E$-dual bundle map by $\rho^{\star}$, i.e.

$$
\rho^{\star}: \operatorname{Hom}(Q, E) \rightarrow \operatorname{Hom}(P, E), \quad \rho^{\star}(\nu)(k)=\nu(\rho(k)), \quad \forall k \in P, \nu \in \operatorname{Hom}(Q, E) .
$$

Definition 2.1. ([5]) An E-Courant algebroid is a quadruple $\left(\mathcal{K},[\cdot, \cdot]_{\mathcal{K}},(\cdot, \cdot)_{E}, \rho\right)$, where $\mathcal{K}$ is a vector bundle over $M$ such that $\left(\Gamma(\mathcal{K}),[\cdot, \cdot]_{\mathcal{K}}\right)$ is a Leibniz algebra, $(\cdot, \cdot)_{E}: \mathcal{K} \otimes \mathcal{K} \rightarrow E$ is a nondegenerate symmetric E-valued pairing, which induces an embedding: $\mathcal{K} \hookrightarrow \operatorname{Hom}(\mathcal{K}, E)$ via $Y(X)=2(X, Y)_{E}$, and $\rho: \mathcal{K} \rightarrow \mathfrak{D E}$ is a bundle map called the anchor, such that for all $X, Y, Z \in \Gamma(\mathcal{K})$, the following properties hold:

$$
\begin{array}{ll}
(\text { EC-1) } & \rho[X, Y]_{\mathcal{K}}=[\rho(X), \rho(Y)]_{\mathfrak{D}} ; \\
(\text { EC-2 }) & {[X, X]_{\mathcal{K}}=\rho^{\star} \mathbb{d}(X, X)_{E} ;} \\
(\text { EC-3) } & \rho(X)(Y, Z)_{E}=\left([X, Y]_{\mathcal{K}}, Z\right)_{E}+\left(Y,[X, Z]_{\mathcal{K}}\right)_{E} ; \\
(\text { EC-4) } & \rho^{\star}(\mathfrak{J} E) \subset \mathcal{K}, \text { i.e. }\left(\rho^{\star}(\mu), X\right)_{E}=\frac{1}{2} \mu(\rho(X)), \forall \mu \in \mathfrak{J} E ; \\
(\text { EC-5) } & \rho \circ \rho^{\star}=0 .
\end{array}
$$


Obviously, a Courant algebroid is an E-Courant algebroid, where $E=M \times \mathbb{R}$, the trivial line bundle. Similar to the proof for Courant algebroids (20, Lemma 2.6.2]), we have

Lemma 2.2. For an $\mathrm{E}$-Courant algebroid $\mathcal{K}$, one has

$$
\left[X, \rho^{\star} \mathrm{d} u\right]_{\mathcal{K}}=2 \rho^{\star} \mathbb{d}\left(X, \rho^{\star} \mathbb{d} u\right)_{E}, \quad\left[\rho^{\star} \mathbb{d} u, X\right]_{\mathcal{K}}=0, \quad \forall X \in \Gamma(\mathcal{K}), u \in \Gamma(E) .
$$

An omni-Lie algebroid, which was introduced in [4, is a very interesting example of E-Courant algebroids. Let us recall it briefly. There is an $E$-valued pairing $(\cdot, \cdot)_{E}$ on $\mathfrak{D} E \oplus \mathfrak{J} E$ defined by

$$
(\mathfrak{d}+\mu, \mathfrak{t}+\nu)_{E}=\frac{1}{2}\left(\langle\mu, \mathfrak{t}\rangle_{E}+\langle\nu, \mathfrak{d}\rangle_{E}\right), \quad \forall \mathfrak{d}+\mu, \mathfrak{t}+\nu \in \mathfrak{D} E \oplus \mathfrak{J} E .
$$

Furthermore, $\Gamma(\mathfrak{J} E)$ is invariant under the Lie derivative $\mathfrak{L}_{\mathfrak{o}}$ for any $\mathfrak{d} \in \Gamma(\mathfrak{D} E)$ which is defined by the Leibniz rule:

$$
\left\langle\mathfrak{L}_{\mathfrak{o}} \mu, \mathfrak{d}^{\prime}\right\rangle_{E} \triangleq \mathfrak{d}\left\langle\mu, \mathfrak{d}^{\prime}\right\rangle_{E}-\left\langle\mu,\left[\mathfrak{d}, \mathfrak{d}^{\prime}\right]_{\mathfrak{D}}\right\rangle_{E}, \quad \forall \mu \in \Gamma(\mathfrak{J} E), \mathfrak{d}^{\prime} \in \Gamma(\mathfrak{D} E) .
$$

On the section space $\Gamma(\mathfrak{D} E \oplus \mathfrak{J} E)$, we can define a bracket as follows

$$
\llbracket \mathfrak{d}+\mu, \mathfrak{r}+\nu \rrbracket \triangleq[\mathfrak{d}, \mathfrak{r}]_{\mathfrak{D}}+\mathfrak{L}_{\mathfrak{d}} \nu-\mathfrak{L}_{\mathfrak{r}} \mu+\mathbb{d} \mu(\mathfrak{r}) .
$$

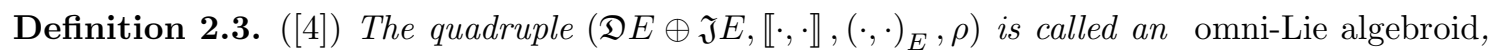
where $\rho$ is the projection from $\mathfrak{D} E \oplus \mathfrak{J} E$ to $\mathfrak{D} E,(\cdot, \cdot)_{E}$ and $\llbracket \cdot, \cdot \rrbracket$ are given by (5) and (6) respectively.

We will denote an omni-Lie algebroid by $\mathfrak{o r}(E)$.

\subsection{Generalized complex structures and generalized contact structures}

The notion of a Courant algebroid was introduced in [17. A Courant algebroid is a quadruple $\left(\mathcal{C}, \llbracket \cdot, \cdot \rrbracket,(\cdot, \cdot)_{+}, \rho\right)$, where $\mathcal{C}$ is a vector bundle over $M, \llbracket \cdot, \cdot \rrbracket$ is a bracket operation on $\Gamma(\mathcal{C}),(\cdot, \cdot)_{+}$ is a nondegenerate symmetric bilinear form on $\mathcal{C}, \rho: \mathcal{C} \longrightarrow T M$ is a bundle map called the anchor, such that some compatibility conditions are satisfied. See 20] for more details. Consider the generalized tangent bundle

$$
\mathbb{T} M:=T M \oplus T^{*} M .
$$

On its section space $\Gamma(\mathbb{T} M)$, there is a Dorfman bracket:

$$
\llbracket X+\xi, Y+\eta \rrbracket=[X, Y]+\mathcal{L}_{X} \eta-i_{Y} \mathrm{~d} \xi, \quad \forall X+\xi, Y+\eta \in \Gamma(\mathbb{T} M) .
$$

Furthermore, there is a canonical nondegenerate symmetric bilinear form on $\mathbb{T} M$ :

$$
(X+\xi, Y+\eta)_{+}=\frac{1}{2}(\eta(X)+\xi(Y)) .
$$

We call $\left(\mathbb{T} M, \llbracket \cdot, \cdot \rrbracket,(\cdot, \cdot)_{+}, \operatorname{pr}_{T M}\right)$ the standard Courant algebroid.

Definition 2.4. A generalized complex structure on a manifold $M$ is a bundle map $\mathcal{J}: \mathbb{T} M \longrightarrow$ $\mathbb{T} M$ satisfying the algebraic properties:

$$
\mathcal{J}^{2}=-\mathrm{id} \quad \text { and } \quad(\mathcal{J}(u), \mathcal{J}(v))_{+}=(u, v)_{+}
$$

and the integrability condition:

$$
\llbracket \mathcal{J}(u), \mathcal{J}(v) \rrbracket-\llbracket u, v \rrbracket-\mathcal{J}(\llbracket \mathcal{J}(u), v \rrbracket+\llbracket u, \mathcal{J}(v) \rrbracket)=0, \quad \forall u, v \in \Gamma(\mathbb{T} M) .
$$

Here, $(\cdot, \cdot)_{+}$and $\llbracket \cdot, \cdot \rrbracket$ are given by (8) and (7) respectively. 
See [8, 9] for more details. Note that only even-dimensional manifolds can have generalized complex structures. In 24], the authors give the odd-dimensional analogue of the concept of a generalized complex structures extending the definition given in [10]. We now recall the definition of a generalized contact bundle from [24]. A generalized contact bundle is a line bundle $L \rightarrow M$ equipped with a generalized contact structure, i.e. a vector bundle endomorphism $\mathcal{I}: \mathfrak{D} L \oplus \mathfrak{J} L \rightarrow$ $\mathfrak{D} L \oplus \mathfrak{J} L$ such that

- $\mathcal{I}$ is almost complex, i.e. $\mathcal{I}^{2}=-\mathrm{id}$,

- $\mathcal{I}$ is skew-symmetric, i.e.

$$
(\mathcal{I} \alpha, \beta)_{L}+(\alpha, \mathcal{I} \beta)_{L}=0, \quad \forall \alpha, \beta \in \Gamma(\mathfrak{D} L \oplus \mathfrak{J} L),
$$

- $\mathcal{I}$ is integrable, i.e.

$$
\llbracket \mathcal{I} \alpha, \mathcal{I} \beta \rrbracket-\llbracket \alpha, \beta \rrbracket-\mathcal{I} \llbracket \mathcal{I} \alpha, \beta \rrbracket-\mathcal{I} \llbracket \alpha, \mathcal{I} \beta \rrbracket=0, \quad \forall \alpha, \beta \in \Gamma(\mathfrak{D} L \oplus \mathfrak{J} L) .
$$

Let $(L \rightarrow M, \mathcal{I})$ be a generalized contact bundle. Using the direct sum oll $(L)=\mathfrak{D} L \oplus \mathfrak{J} L$ and the definition, one can see that

$$
\mathcal{I}=\left(\begin{array}{cc}
\phi & J^{\sharp} \\
\omega_{b} & -\phi^{\dagger}
\end{array}\right),
$$

where $J$ is a Jacobi bi-derivation, $\phi$ is an endomorphism of $\mathfrak{D} L$ compatible with $J$, and the 2form $\omega: \wedge^{2} \mathfrak{D} L \rightarrow L$ and its associated vector bundle morphism $\omega_{b}: \mathfrak{D} L \rightarrow \mathfrak{J} L$ satisfy additional compatibility conditions [24].

\section{VB-Courant algebroids and E-Courant algebroids}

In this section, we highlight the relation between VB-Courant algebroids and E-Courant algebroids and give more examples of E-Courant algebroids.

Denote a double vector bundle

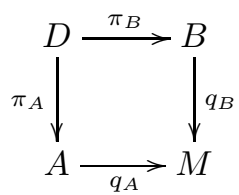

with core $C$ by $(D ; A, B ; M)$. The space of sections $\Gamma_{B}(D)$ is generated as a $C^{\infty}(B)$-module by core sections $\Gamma_{B}^{c}(D)$ and linear sections $\Gamma_{B}^{l}(D)$. See [19] for more details. For a section $c: M \rightarrow C$, the corresponding core section $c^{\dagger}: B \rightarrow D$ is defined as

$$
c^{\dagger}\left(b_{m}\right)=\tilde{0}_{b_{m}}+{ }_{A} \overline{c(m)}, \quad \forall m \in M, b_{m} \in B_{m},
$$

where ${ }^{-}$means the inclusion $C \hookrightarrow D$. A section $\xi: B \rightarrow D$ is called linear if it is a bundle morphism from $B \rightarrow M$ to $D \rightarrow A$ over a section $a \in \Gamma(A)$. Given $\psi \in \Gamma\left(B^{*} \otimes C\right)$, there is a linear section $\tilde{\psi}: B \rightarrow D$ over the zero section $0^{A}: M \rightarrow A$ given by

$$
\tilde{\psi}\left(b_{m}\right)=\tilde{0}_{b_{m}}+{ }_{A} \overline{\psi\left(b_{m}\right)} .
$$

Note that $\Gamma_{B}^{l}(D)$ is locally free as a $C^{\infty}(M)$-module. Therefore, $\Gamma_{B}^{l}(D)$ is equal to $\Gamma(\hat{A})$ for some vector bundle $\hat{A} \rightarrow M$. Moreover, we have the following short exact sequence of vector bundles over $M$

$$
0 \rightarrow B^{*} \otimes C \longrightarrow \hat{A} \longrightarrow A \rightarrow 0 \text {. }
$$


Example 3.1. Let $E$ be a vector bundle over $M$.

(a) The tangent bundle $(T E ; T M, E ; M)$ is a double vector bundle with core $E$. Then $\hat{A}$ is the gauge bundle $\mathfrak{D} E$ and the exact sequence (9) is exactly the Atiyah sequence (1).

(b) The cotangent bundle $\left(T^{*} E ; E^{*}, E ; M\right)$ is a double vector bundle with core $T^{*} M$. In this case, $\hat{A}$ is exactly the jet bundle $\mathfrak{J} E^{*}$ and the exact sequence (9) is indeed the jet sequence (3).

Definition 3.2. ([15]) A VB-Courant algebroid is a metric double vector bundle

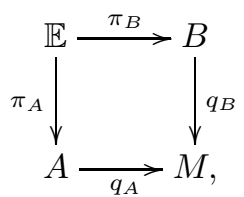

with core $C$ such that $\mathbb{E} \rightarrow B$ is a Courant algebroid and the following conditions are satisfied:

(i) The anchor map $\Theta: \mathbb{E} \rightarrow T B$ is linear. That is

$$
\Theta:(\mathbb{E} ; A, B ; M) \longrightarrow(T B ; T M, B ; M)
$$

is a morphism of double vector bundles.

(ii) The Courant bracket is linear. That is

$$
\llbracket \Gamma_{B}^{l}(\mathbb{E}), \Gamma_{B}^{l}(\mathbb{E}) \rrbracket \subseteq \Gamma_{B}^{l}(\mathbb{E}), \quad \llbracket \Gamma_{B}^{l}(\mathbb{E}), \Gamma_{B}^{c}(\mathbb{E}) \rrbracket \subseteq \Gamma_{B}^{c}(\mathbb{E}), \quad \llbracket \Gamma_{B}^{c}(\mathbb{E}), \Gamma_{B}^{c}(\mathbb{E}) \rrbracket=0 .
$$

For a VB-Courant algebroid $\mathbb{E}$, we have the exact sequence (9). Note that the restriction of the pairing on $\mathbb{E}$ to linear sections of $\mathbb{E}$ defines a nondegenerate pairing on $\hat{A}$ with values in $B^{*}$, which is guaranteed by the metric double vector bundle structure; see [11. Coupled with the fact that the Courant bracket is closed on linear sections, one gets the following result.

Proposition 3.3. (11]) The vector bundle $\hat{A}$ inherits a Courant algebroid structure with the pairing taking values in $B^{*}$, which is called the fat Courant algebroid of this VB-Courant algebroid.

Alternatively, we have

Proposition 3.4. For a VB-Courant algebroid $(\mathbb{E} ; A, B ; M)$, its associated fat Courant algebroid is a $B^{*}$-Courant algebroid.

Example 3.5. (Standard VB-Courant algebroid over a vector bundle) For a vector bundle $E$, there is a standard VB-Courant algebroid

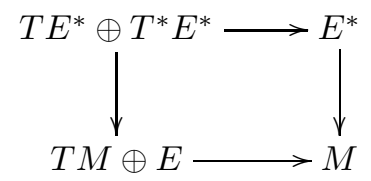

with base $E^{*}$ and core $E^{*} \oplus T^{*} M \rightarrow M$. The corresponding exact sequence is given by

$$
0 \rightarrow \operatorname{gl}(E) \oplus T^{*} M \otimes E \longrightarrow \hat{A} \longrightarrow T M \oplus E \rightarrow 0 .
$$

Actually, by Example 3.1, the corresponding fat Courant algebroid $\hat{A}$ here is exactly the omniLie algebroid $\mathfrak{o r}(E)=\mathfrak{D} E \oplus \mathfrak{J} E$. So the omni-Lie algebroid is the linearization of the standard VB-Courant algebroid. 
Example 3.6. (Tangent VB-Courant algebroid) The tangent bundle $T C$ of a Courant algebroid $\mathcal{C} \rightarrow M$

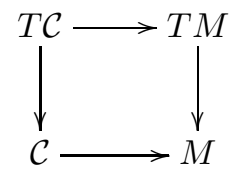

carries a VB-Courant algebroid structure with base $T M$ and core $\mathcal{C} \rightarrow M$. The associated exact sequence is

$$
0 \rightarrow T^{*} M \otimes \mathcal{C} \longrightarrow \hat{\mathcal{C}} \longrightarrow \mathcal{C} \rightarrow 0 .
$$

Actually the fat Courant algebroid $\hat{\mathcal{C}}$ is $\mathfrak{J C}$, which is a $T^{*} M$-Courant algebroid by Proposition 3.4. So we get that on the jet bundle of a Courant algebroid, there is a $T^{*} M$-Courant algebroid structure. This result was first given in [5].

A crossed module of Lie algebras consists of a pair of Lie algebras $(\mathfrak{m}, \mathfrak{g})$, an action $\triangleright$ of $\mathfrak{g}$ on $\mathfrak{m}$ and a Lie algebra morphism $\phi: \mathfrak{m} \rightarrow \mathfrak{g}$ such that

$$
\phi(\xi) \triangleright \eta=[\xi, \eta]_{\mathfrak{m}}, \quad \phi(x \triangleright \xi)=[x, \phi(\xi)]_{\mathfrak{g}}, \quad \forall x \in \mathfrak{g}, \quad \xi, \eta \in \mathfrak{m} .
$$

Given a crossed module, there is an action $\rho: \mathfrak{g} \ltimes \mathfrak{g}^{*} \longrightarrow \mathfrak{X}\left(\mathfrak{m}^{*}\right)$ of the natural quadratic Lie algebra $\mathfrak{g} \ltimes \mathfrak{g}^{*}$ on $\mathfrak{m}^{*}$ given by

$$
\rho(u+\alpha)=u \triangleright \cdot+\phi^{*} \alpha,
$$

where $u \triangleright \cdot \in \operatorname{gl}(\mathfrak{m})$ is viewed as a linear vector field on $\mathfrak{m}^{*}$ and $\phi^{*} \alpha \in \mathfrak{m}^{*}$ is viewed as a constant vector field on $\mathfrak{m}^{*}$. Note that this action is coisotropic. We get the action Courant algebroid [16] $\left(\mathfrak{g} \ltimes \mathfrak{g}^{*}\right) \times \mathfrak{m}^{*}$ over $\mathfrak{m}^{*}$ with the anchor given by $\rho$ and the Dorfman bracket given by

$$
\left[e_{1}, e_{2}\right]=\mathcal{L}_{\rho\left(e_{1}\right)} e_{2}-\mathcal{L}_{\rho\left(e_{2}\right)} e_{1}+\left[e_{1}, e_{2}\right]_{\mathfrak{g} \ltimes \mathfrak{g}^{*}}+\rho^{*}\left\langle\mathrm{~d} e_{1}, e_{2}\right\rangle .
$$

for any $e_{1}, e_{2} \in \Gamma\left(\left(\mathfrak{g} \ltimes \mathfrak{g}^{*}\right) \times \mathfrak{m}^{*}\right)$. Here $\mathrm{d} e_{1} \in \Omega^{1}\left(\mathfrak{m}^{*}, \mathfrak{g} \ltimes \mathfrak{g}^{*}\right)$ is given by Lie derivatives: $\left(\mathrm{d} e_{1}\right)(X)=$ $\mathcal{L}_{X} e_{1}$ for $X \in \mathfrak{X}\left(\mathfrak{m}^{*}\right)$. Moreover, it is a VB-Courant algebroid

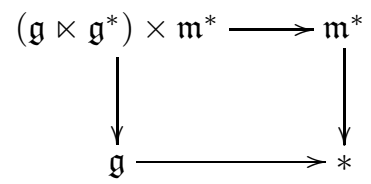

with base $\mathfrak{m}^{*}$ and core $\mathfrak{g}^{*}$. See [15] for details. The associated exact sequence is

$$
0 \rightarrow \mathfrak{m} \otimes \mathfrak{g}^{*} \cong \operatorname{Hom}(\mathfrak{g}, \mathfrak{m}) \longrightarrow \hat{A} \longrightarrow \mathfrak{g} \rightarrow 0 .
$$

Since the double vector bundle is trivial, we have $\hat{A}=\operatorname{Hom}(\mathfrak{g}, \mathfrak{m}) \oplus \mathfrak{g}$.

Moreover, applying (10), we get the $\operatorname{Dorfman}$ bracket on $\operatorname{Hom}(\mathfrak{g}, \mathfrak{m}) \oplus \mathfrak{g}$.

Proposition 3.7. With the above notations, $\left(\operatorname{Hom}(\mathfrak{g}, \mathfrak{m}) \oplus \mathfrak{g},[\cdot, \cdot],(\cdot, \cdot)_{\mathfrak{m}}, \rho=0\right)$ is an $\mathfrak{m}$-Courant algebroid, where the pairing $(\cdot, \cdot)_{\mathfrak{m}}$ is given by

$$
(A+u, B+v)_{\mathfrak{m}}=\frac{1}{2}(A v+B u),
$$

and the Dorfman bracket is given by

$$
\begin{aligned}
{[u, v] } & =[u, v]_{\mathfrak{g}} ; \\
{[A, B] } & =A \circ \phi \circ B-B \circ \phi \circ A ; \\
{[A, v] } & =A \circ \operatorname{ad}_{v}^{0}-\operatorname{ad}_{v}^{1} \circ A+\cdot \triangleright A v+\phi(A v) ; \\
{[v, A] } & =\operatorname{ad}_{v}^{1} \circ A-A \circ \operatorname{ad}_{v}^{0}
\end{aligned}
$$


for all $A, B \in \operatorname{Hom}(\mathfrak{g}, \mathfrak{m}), u, v \in \mathfrak{g}$. Here $\operatorname{ad}_{v}^{0} \in \operatorname{gl}(\mathfrak{g})$ and $\operatorname{ad}_{v}^{1} \in \operatorname{gl}(\mathfrak{m})$ are given by $\operatorname{ad}_{v}^{0}(u)=[v, u]_{\mathfrak{g}}$ and $\operatorname{ad}_{v}^{1}(a)=v \triangleright$ a respectively and $\cdot \triangleright A v \in \operatorname{Hom}(\mathfrak{g}, \mathfrak{m})$ is defined by $(\cdot \triangleright A v)(u)=u \triangleright A v$.

Proof. By (10), it is obvious that

$$
[u, v]=[u, v]_{\mathfrak{g}} .
$$

For $A, B \in \operatorname{Hom}(\mathfrak{g}, \mathfrak{m}), v \in \mathfrak{g}$, applying (10), we find

$$
[A, B]=\mathcal{L}_{\rho(A)} B-\mathcal{L}_{\rho(B)} A=\rho(A) B-\rho(B) A=A \circ \phi \circ B-B \circ \phi \circ A .
$$

Observe that $\mathcal{L}_{\rho(v)} A=\operatorname{ad}_{v}^{1}(A)=\operatorname{ad}_{v}^{1} \circ A$ and $[A, v]_{\mathfrak{g} \ltimes \mathfrak{g}^{*}}=-\left(\operatorname{ad}^{0}\right)_{v}^{*} A=A \circ \operatorname{ad}_{v}^{0}$. We have

$$
\begin{aligned}
{[A, v] } & =\mathcal{L}_{\rho(A)} v-\mathcal{L}_{\rho(v)} A+[A, v]_{\mathfrak{g} \ltimes \mathfrak{g}^{*}}+\rho^{*}\langle\mathrm{~d} A, v\rangle \\
& =0-\operatorname{ad}_{v}^{1} \circ A+A \circ \operatorname{ad}_{v}^{0}+\cdot \triangleright A v+\phi(A v),
\end{aligned}
$$

where we have used

$$
\rho^{*}\langle\mathrm{~d} A, v\rangle(u+B)=\rho(u+B)(A v)=u \triangleright A v+B(\phi(A v)) .
$$

Finally, we have

$$
\begin{aligned}
{[v, A] } & =\mathcal{L}_{\rho(v)} A-\mathcal{L}_{\rho(A)} v+[v, A]_{\mathfrak{g} \ltimes \mathfrak{g}^{*}}+\rho^{*}\langle\mathrm{~d} v, A\rangle \\
& =\operatorname{ad}_{v}^{1} \circ A+0-A \circ \operatorname{ad}_{v}^{0}+0 .
\end{aligned}
$$

This completes the proof.

Remark 3.8. This bracket can be viewed as a generalization of an omni-Lie algebra. See [13, Example 5.2] for more details.

More generally, since the category of Lie 2-algebroids and the category of VB-Courant algebroids are equivalent (see [15]), we get an E-Courant algebroid from a Lie 2-algebroid. This construction first appeared in [11, Corollary 6.9]. Explicitly, let $\left(A_{0} \oplus A_{-1}, \rho_{A_{0}}, l_{1}, l_{2}=l_{2}^{0}+l_{2}^{1}, l_{3}\right)$ be a Lie 2-algebroid. Then we have an $A_{-1}$-Courant algebroid structure on

$$
\operatorname{Hom}\left(A_{0}, A_{-1}\right) \oplus A_{0},
$$

where the paring is given by

$$
\left(D+u, D^{\prime}+v\right)_{A_{-1}}=\frac{1}{2}\left(D v+D^{\prime} u\right), \quad \forall D, D^{\prime} \in \Gamma\left(\operatorname{Hom}\left(A_{0}, A_{-1}\right)\right), u, v \in \Gamma\left(A_{0}\right)
$$

the anchor is

$$
\rho: \operatorname{Hom}\left(A_{0}, A_{-1}\right) \oplus A_{0} \rightarrow \mathfrak{D} A_{-1}, \quad \rho(D+u)=D \circ l_{1}+l_{2}^{1}(u, \cdot),
$$

and the Dorfman bracket is given by

$$
\begin{aligned}
{[u, v] } & =l_{2}^{0}(u, v)+l_{3}(u, v, \cdot) \\
{\left[D, D^{\prime}\right] } & =D \circ l_{1} \circ D^{\prime}-D^{\prime} \circ l_{1} \circ D \\
{[D, v] } & =-l_{2}^{1}(v, D(\cdot))+D\left(l_{2}^{0}(v, \cdot)\right)+l_{2}^{1}(\cdot, D(v))+l_{1}(D(v)) \\
{[v, D] } & =l_{2}^{1}(v, D(\cdot))-D\left(l_{2}^{0}(v, \cdot)\right)
\end{aligned}
$$




\section{Generalized complex structures on E-Courant algebroids}

In this section, we introduce the notion of a generalized complex structure on an E-Courant algebroid. We will see that it unifies the usual generalized complex structure on an even-dimensional manifold and the generalized contact structure on an odd-dimensional manifold.

Definition 4.1. A bundle map $\mathcal{J}: \mathcal{K} \longrightarrow \mathcal{K}$ is called a generalized almost complex structure on an $\mathrm{E}$-Courant algebroid $\left(\mathcal{K},[\cdot, \cdot]_{\mathcal{K}},(\cdot, \cdot)_{E}, \rho\right)$ if it satisfies the algebraic properties

$$
\mathcal{J}^{2}=-1 \quad \text { and } \quad(\mathcal{J}(X), \mathcal{J}(Y))_{E}=(X, Y)_{E} .
$$

Furthermore, $\mathcal{J}$ is called a generalized complex structure if the following integrability condition is satisfied:

$$
[\mathcal{J}(X), \mathcal{J}(Y)]_{\mathcal{K}}-[X, Y]_{\mathcal{K}}-\mathcal{J}\left([\mathcal{J}(X), Y]_{\mathcal{K}}+[X, \mathcal{J}(Y)]_{\mathcal{K}}\right)=0, \quad \forall X, Y \in \Gamma(\mathcal{K}) .
$$

Proposition 4.2. Let $\mathcal{J}: \mathcal{K} \longrightarrow \mathcal{K}$ be a generalized almost complex structure on an $\mathrm{E}$-Courant algebroid $\left(\mathcal{K},[\cdot, \cdot]_{\mathcal{K}},(\cdot, \cdot)_{E}, \rho\right)$. Then we have

$$
\left.\mathcal{J}^{\star}\right|_{\mathcal{K}}=-\mathcal{J}
$$

Proof. By (11), for all $X, Y \in \Gamma(\mathcal{K})$, we have

$$
\mathcal{J}^{\star}(\mathcal{J}(Y))(X)=\mathcal{J}(Y)(\mathcal{J}(X))=2(\mathcal{J}(X), \mathcal{J}(Y))_{E}=2(X, Y)_{E}=Y(X) .
$$

Since $X \in \Gamma(\mathcal{K})$ is arbitrary, we have

$$
\mathcal{J}^{\star}(\mathcal{J}(Y))=Y, \quad \forall Y \in \Gamma(\mathcal{K}) .
$$

For any $Z \in \Gamma(\mathcal{K})$, let $Y=-\mathcal{J}(Z)$. By (11), we have $Z=\mathcal{J}(Y)$. Then we have

$$
\mathcal{J}^{\star}(Z)=\mathcal{J}^{\star}(\mathcal{J}(Y))=Y=-\mathcal{J}(Z),
$$

which implies that $\left.\mathcal{J}^{\star}\right|_{\mathcal{K}}=-\mathcal{J}$.

Remark 4.3. Generalized complex structures on an $\mathrm{E}$-Courant algebroid $\left(\mathcal{K},[\cdot, \cdot]_{\mathcal{K}},(\cdot, \cdot)_{E}, \rho\right)$ are in one-to-one correspondence with Dirac sub-bundles $S \subset \mathcal{K} \otimes \mathbb{C}$ such that $\mathcal{K} \otimes \mathbb{C}=S \oplus \bar{S}$. By a Dirac sub-bundle of $\mathcal{K}$, we mean a sub-bundle $S \subset \mathcal{K}$ which is closed under the bracket $[\cdot, \cdot]_{\mathcal{K}}$ and satisfies $S=S^{\perp}$. The pair $(S, \bar{S})$ is an E-Lie bialgebroid in the sense of [5].

Remark 4.4. Obviously, the notion of a generalized contact bundle associated to L, which was introduced in [24], is a special case of Definition 4.1 where $E$ is the line bundle L. In particular, if $E$ is the trivial line bundle $L^{\circ}=M \times \mathbb{R}$, we have

$$
\mathfrak{D} L^{\circ}=T M \oplus \mathbb{R}, \quad \mathfrak{J} L^{\circ}=T^{*} M \oplus \mathbb{R} .
$$

Therefore, $\mathcal{E}^{1}(M)=\mathfrak{D} L^{\circ} \oplus \mathfrak{J} L^{\circ}$. Thus, a generalized complex structure on an E-Courant algebroid unifies generalized complex structures on even-dimensional manifolds and generalized contact bundles on odd-dimensional manifolds

Example 4.5. Consider the E-Courant algebroid $A^{*} \otimes E \oplus A$ given in [5, Example 2.9] for any Lie algebroid $\left(A,[\cdot, \cdot]_{A}, a\right)$ and an $A$-module $E$. Twisted by a 3-cocycle $\Theta \in \Gamma\left(\wedge^{3} A^{*}, E\right)$, one obtains the AV-Courant algebroid introduced in [14] by Li-Bland. Consider $\mathcal{J}$ of the form $\mathcal{J}_{D}=\left(\begin{array}{cc}-R_{D} & 0 \\ 0 & D\end{array}\right)$, where $D \in \operatorname{gl}(A)$ and $R_{D}: A^{*} \otimes E \longrightarrow A^{*} \otimes E$ is given by $R_{D}(\phi)=\phi \circ D$. We get that $\mathcal{J}$ is a 
generalized complex structure on the E-Courant algebroid $A^{*} \otimes E \oplus A$ if and only if $D$ is a Nijenhuis operator on the Lie algebroid $A$ and $D^{2}=-1$.

Actually, $D^{2}=-1$ ensures that condition (11) holds. The Dorfman bracket on $\mathcal{K}=A^{*} \otimes E \oplus A$ is given by

$$
[u+\Phi, v+\Psi]_{\mathcal{K}}=[u, v]_{A}+\mathcal{L}_{u} \Psi-\mathcal{L}_{v} \Phi+\rho^{\star} \mathbb{d} \Phi(v), \quad \forall u, v \in \Gamma(A), \Phi, \Psi \in \Gamma\left(A^{*} \otimes E\right),
$$

where $\rho^{\star}: \mathfrak{J} E \rightarrow A^{*} \otimes E$ is the dual of the $A$-action $\rho: A \rightarrow \mathfrak{D} E$ on $E$. Then it is straightforward to see that the integrability condition (12) holds if and only if $D$ is a Nijenhuis operator on $A$.

Any generalized complex structure on a Courant algebroid induces a Poisson structure on the base manifold (see e.g. 1]). Similarly, any generalized complex structure on an E-Courant algebroid induces a Lie algebroid or a local Lie algebra structure ([12]) on $E$.

Theorem 4.6. Let $\mathcal{J}: \mathcal{K} \longrightarrow \mathcal{K}$ be a generalized complex structure on an $\mathrm{E}$-Courant algebroid $\left(\mathcal{K},[\cdot, \cdot]_{\mathcal{K}},(\cdot, \cdot)_{E}, \rho\right)$. Define a bracket operation $[\cdot, \cdot]_{E}: \Gamma(E) \wedge \Gamma(E) \longrightarrow \Gamma(E)$ by

$$
[u, v]_{E} \triangleq 2\left(\mathcal{J} \rho^{\star} \mathbb{d} u, \rho^{\star} \mathbb{d} v\right)_{E}=\left(\rho \circ \mathcal{J} \circ \rho^{\star}\right)(\mathbb{d} u)(v), \quad \forall u, v \in \Gamma(E) .
$$

Then $\left(E,[\cdot, \cdot]_{E}, \dot{\mathfrak{j}} \circ \rho \circ \mathcal{J} \circ \rho^{\star} \circ \mathbb{d}\right)$ is a Lie algebroid when $\operatorname{rank}(E) \geq 2$ and $\left(E,[\cdot, \cdot]_{E}\right)$ is a local Lie algebra when $\operatorname{rank}(E)=1$.

Proof. The bracket is obviously skew-symmetric. By the integrability of $\mathcal{J}$, we have

$$
\left[\mathcal{J}\left(\rho^{\star} \mathrm{d} u\right), \mathcal{J}\left(\rho^{\star} \mathrm{d} v\right)\right]_{\mathcal{K}}-\left[\rho^{\star} \mathrm{d} u, \rho^{\star} \mathrm{d} v\right]_{\mathcal{K}}-\mathcal{J}\left(\left[\mathcal{J}\left(\rho^{\star} \mathrm{d} u\right), \rho^{\star} \mathrm{d} v\right]_{\mathcal{K}}+\left[\rho^{\star} \mathrm{d} u, \mathcal{J}\left(\rho^{\star} \mathrm{d} v\right)\right]_{\mathcal{K}}\right)=0 .
$$

Pairing with $\rho^{\star} \mathrm{d} w$ for $w \in \Gamma(E)$, by (EC-3) in Definition 2.1 and the first equation in Lemma 2.2. we have

$$
\begin{aligned}
& \left(\left[\mathcal{J}\left(\rho^{\star} \mathrm{d} u\right), \mathcal{J}\left(\rho^{\star} \mathrm{d} v\right)\right]_{\mathcal{K}}, \rho^{\star} \mathrm{d} w\right)_{E} \\
= & \rho\left(\mathcal{J} \rho^{\star} \mathrm{d} u\right)\left(\mathcal{J} \rho^{\star} \mathrm{d} v, \rho^{\star} \mathrm{d} w\right)_{E}-\left(\mathcal{J} \rho^{\star} \mathrm{d} v,\left[\mathcal{J} \rho^{\star} \mathrm{d} u, \rho^{\star} \mathrm{d} w\right]_{\mathcal{K}}\right)_{E} \\
= & 2\left(\rho^{\star} \mathrm{d}\left(\mathcal{J} \rho^{\star} \mathrm{d} v, \rho^{\star} \mathrm{d} w\right)_{E}, \mathcal{J} \rho^{\star} \mathrm{d} u\right)_{E}-2\left(\mathcal{J} \rho^{\star} \mathrm{d} v, \rho^{\star} \mathrm{d}\left(\mathcal{J} \rho^{\star} \mathrm{d} u, \rho^{\star} \mathrm{d} w\right)_{E}\right)_{E} \\
= & \frac{1}{2}\left[u,[v, w]_{E}\right]_{E}-\frac{1}{2}\left[v,[u, w]_{E}\right]_{E} .
\end{aligned}
$$

By (EC-1) and (EC-5) in Definition 2.1, we have

$$
\left(\left[\rho^{\star} \mathrm{d} u, \rho^{\star} \mathrm{d} v\right]_{\mathcal{K}}, \rho^{\star} \mathrm{d} w\right)_{E}=0 .
$$

Finally, using Lemma 2.2, we have

$$
\begin{aligned}
& \left.\left(\left[\mathcal{J}\left(\rho^{\star} \mathrm{d} u\right), \rho^{\star} \mathrm{d} v\right]_{\mathcal{K}}+\left[\rho^{\star} \mathrm{d} u, \mathcal{J}\left(\rho^{\star} \mathrm{d} v\right)\right]_{\mathcal{K}}\right), \mathcal{J} \rho^{\star} \mathrm{d} w\right)_{E} \\
= & 2\left(\rho^{\star} \mathrm{d}\left(\mathcal{J} \rho^{\star} \mathrm{d} u, \rho^{\star} \mathrm{d} v\right)_{E}, \mathcal{J} \rho^{\star} \mathrm{d} w\right)_{E}+0 \\
= & \frac{1}{2}\left[w,[u, v]_{E}\right]_{E} .
\end{aligned}
$$

Thus we get the Jacobi identity for $[\cdot, \cdot]_{E}$. To see the Leibniz rule, by definition, we have

$$
[u, f v]_{E}=f[u, v]_{E}+\dot{\mathfrak{j}} \rho \mathcal{J} \rho^{\star} \mathbb{d}(u)(f) v .
$$

So it is a Lie algebroid structure if and only if $j \circ \rho \circ \mathcal{J} \circ \rho^{\star} \circ \mathbb{d}: E \rightarrow T M$ is a bundle map, which is always true when $\operatorname{rank}(E) \geq 2$ (see the proof of [4, Theorem 3.11]). 


\section{$5 \quad$ Generalized complex structures on omni-Lie algebroids}

In this section, we study generalized complex structures on the omni-Lie algebroid ol $(E)$. We view $\mathfrak{o r}(E)$ as a sub-bundle of $\operatorname{Hom}(\mathfrak{o r}(E), E)$ by the nondegenerate $E$-valued pairing $(\cdot, \cdot)_{E}$, i.e.

$$
e_{2}\left(e_{1}\right) \triangleq 2\left(e_{1}, e_{2}\right)_{E}, \quad \forall e_{1}, e_{2} \in \Gamma(\mathfrak{o r}(E)) .
$$

By Proposition 4.2, we have

Corollary 5.1. A bundle map $\mathcal{J}: \mathfrak{o l}(E) \longrightarrow \mathfrak{o l}(E)$ is a generalized almost complex structure on the omni-Lie algebroid ol $(E)$ if and only if the following conditions are satisfied:

$$
\mathcal{J}^{2}=-\mathrm{id}, \quad \mathcal{J}^{\star} \operatorname{lor}(E)=-\mathcal{J} .
$$

Since $\mathfrak{o l}(E)$ is the direct sum of $\mathfrak{D} E$ and $\mathfrak{J} E$, we can write a generalized almost complex structure $\mathcal{J}$ in the form of a matrix. To do that, we need some preparations.

Vector bundles $\operatorname{Hom}\left(\wedge^{k} \mathfrak{D} E, E\right)_{\mathfrak{J} E}$ and $\operatorname{Hom}\left(\wedge^{k} \mathfrak{J} E, E\right)_{\mathfrak{D} E}$ are introduced in [5] and 21] to study deformations of omni-Lie algebroids and deformations of Lie algebroids respectively. More precisely, we have

$$
\begin{aligned}
& \operatorname{Hom}\left(\wedge^{k} \mathfrak{D} E, E\right)_{\mathfrak{J} E} \triangleq\left\{\mu \in \operatorname{Hom}\left(\wedge^{k} \mathfrak{D} E, E\right) \mid \operatorname{Im}\left(\mu_{\natural}\right) \subset \mathfrak{J} E\right\}, \quad(k \geq 2), \\
& \operatorname{Hom}\left(\wedge^{k} \mathfrak{J} E, E\right)_{\mathfrak{D} E} \triangleq\left\{\mathfrak{d} \in \operatorname{Hom}\left(\wedge^{k} \mathfrak{J} E, E\right) \mid \operatorname{Im}\left(\mathfrak{d}^{\sharp}\right) \subset \mathfrak{D} E\right\}, \quad(k \geq 2),
\end{aligned}
$$

in which $\mu_{\natural}: \wedge^{k-1} \mathfrak{D} E \longrightarrow \operatorname{Hom}(\mathfrak{D} E, E)$ is given by

$$
\mu_{\natural}\left(\mathfrak{d}_{1}, \cdots, \mathfrak{d}_{k-1}\right)\left(\mathfrak{d}_{k}\right)=\mu\left(\mathfrak{d}_{1}, \cdots, \mathfrak{d}_{k-1}, \mathfrak{d}_{k}\right), \quad \forall \mathfrak{d}_{1}, \cdots, \mathfrak{d}_{k} \in \mathfrak{D} E,
$$

and $\mathfrak{d}^{\sharp}$ is defined similarly. By (2) , for any $\mu \in \operatorname{Hom}\left(\wedge^{k} \mathfrak{D} E, E\right)_{\mathfrak{J} E}$, we have

$$
\mu\left(\mathfrak{d}_{1}, \cdots, \mathfrak{d}_{k-1}, \Phi\right)=\Phi \circ \mu\left(\mathfrak{d}_{1}, \cdots, \mathfrak{d}_{k-1}, \mathrm{id}_{E}\right) .
$$

Furthermore, $\left(\Gamma\left(\operatorname{Hom}\left(\wedge^{\bullet} \mathfrak{D} E, E\right)_{\mathfrak{J} E}\right), \mathbb{d}\right)$ is a subcomplex of $\left(\Gamma\left(\operatorname{Hom}\left(\wedge^{\bullet} \mathfrak{D} E, E\right)\right.\right.$, d $)$, where $\mathbb{d}$ is the coboundary operator of the gauge Lie algebroid $\mathfrak{D} E$ with the obvious action on $E$.

Proposition 5.2. Any generalized almost complex structure $\mathcal{J}$ on the omni-Lie algebroid or( $E)$ must be of the form

$$
\mathcal{J}=\left(\begin{array}{cc}
N & \pi^{\sharp} \\
\sigma_{\natural} & -N^{\star}
\end{array}\right),
$$

where $N: \mathfrak{D} E \longrightarrow \mathfrak{D} E$ is a bundle map satisfying $N^{\star}(\mathfrak{J} E) \subset \mathfrak{J} E, \pi \in \Gamma\left(\operatorname{Hom}\left(\wedge^{2} \mathfrak{J} E, E\right)_{\mathfrak{D} E}\right)$, $\sigma \in \Gamma\left(\operatorname{Hom}\left(\wedge^{2} \mathfrak{D} E, E\right)_{\mathfrak{J} E}\right)$ such that the following conditions hold:

$$
\pi^{\sharp} \circ \sigma_{\natural}+N^{2}=-\mathrm{id}, \quad N \circ \pi^{\sharp}=\pi^{\sharp} \circ N^{\star}, \quad \sigma_{\natural} \circ N=N^{\star} \circ \sigma_{\natural} .
$$

Proof. By Corollary [5.1, for any generalized almost complex structure $\mathcal{J}$, we have $\left.\mathcal{J}^{\star}\right|_{\text {ol }(E)}=-\mathcal{J}$. Thus $\mathcal{J}$ must be of the form

$$
\mathcal{J}=\left(\begin{array}{cc}
N & \phi \\
\psi & -N^{\star}
\end{array}\right)
$$

where $N: \mathfrak{D} E \longrightarrow \mathfrak{D} E$ is a bundle map satisfying $N^{\star}(\mathfrak{J} E) \subset \mathfrak{J} E, \phi: \mathfrak{J} E \longrightarrow \mathfrak{D} E$ and $\psi: \mathfrak{D} E \longrightarrow$ $\mathfrak{J} E$ are bundle maps satisfying

$$
-(\phi(\mu), \nu)_{E}=(\mu, \phi(\nu))_{E}, \quad-(\psi(\mathfrak{d}), \mathfrak{t})_{E}=(\mathfrak{d}, \psi(\mathfrak{t}))_{E} .
$$

Therefore, we have $\phi=\pi^{\sharp}$, for some $\pi \in \Gamma\left(\operatorname{Hom}\left(\wedge^{2} \mathfrak{J} E, E\right)_{\mathfrak{D} E}\right)$, and $\psi=\sigma_{\natural}$ for some $\sigma \in$ $\Gamma\left(\operatorname{Hom}\left(\wedge^{2} \mathfrak{D} E, E\right)_{\mathfrak{J} E}\right)$. This finishes the proof of the first part. As for the second part, it is straightforward to see that the conditions follow from the fact that $\mathcal{J}^{2}=-$ id. 
Remark 5.3. For a line bundle $L$, we have $\mathfrak{J} L=\operatorname{Hom}(\mathfrak{D} L, L)$ and $\mathfrak{D} L=\operatorname{Hom}(\mathfrak{J} L, L)$. Therefore, the condition $N^{\star}(\mathfrak{J} L) \subset \mathfrak{J} L$ always holds.

Theorem 5.4. A generalized almost complex structure $\mathcal{J}$ given by (16) is a generalized complex structure on the omni-Lie algebroid ol $(E)$ if and only if

(i) $\pi$ satisfies the equation:

$$
\pi^{\sharp}\left([\mu, \nu]_{\pi}\right)=\left[\pi^{\sharp}(\mu), \pi^{\sharp}(\nu)\right]_{\mathfrak{D}}, \quad \forall \mu, \nu \in \Gamma(\mathfrak{J} E),
$$

where the bracket $[\cdot, \cdot]_{\pi}$ on $\Gamma(\mathfrak{J} E)$ is defined by

$$
[\mu, \nu]_{\pi} \triangleq \mathfrak{L}_{\pi^{\sharp}(\mu)} \nu-\mathfrak{L}_{\pi^{\sharp}(\nu)} \mu-\mathbb{d}\left\langle\pi^{\sharp}(\mu), \nu\right\rangle_{E} .
$$

(ii) $\pi$ and $N$ are related by the following formula:

$$
N^{\star}\left([\mu, \nu]_{\pi}\right)=\mathfrak{L}_{\pi^{\sharp}(\mu)}\left(N^{\star}(\nu)\right)-\mathfrak{L}_{\pi^{\sharp}(\nu)}\left(N^{\star}(\mu)\right)-\mathbb{d} \pi\left(N^{\star}(\mu), \nu\right) .
$$

(iii) $N$ satisfies the condition:

$$
T(N)(\mathfrak{d}, \mathfrak{t})=\pi^{\sharp}\left(i_{\mathfrak{d} \wedge} \mathfrak{t} \mathbb{d} \sigma\right), \quad \forall \mathfrak{d}, \mathfrak{t} \in \Gamma(\mathfrak{D} E),
$$

where $T(N)$ is the Nijenhuis tensor of $N$ defined by

$$
T(N)(\mathfrak{d}, \mathfrak{t})=[N(\mathfrak{d}), N(\mathfrak{t})]_{\mathfrak{D}}-N\left([N(\mathfrak{d}), \mathfrak{t}]_{\mathfrak{D}}+[\mathfrak{d}, N(\mathfrak{t})]_{\mathfrak{D}}-N[\mathfrak{d}, \mathfrak{t}]_{\mathfrak{D}}\right) .
$$

(iv) $N$ and $\sigma$ are related by the following condition

$$
\mathbb{d} \sigma(N(\mathfrak{d}), \mathfrak{t}, \mathfrak{k})+\mathbb{d} \sigma(\mathfrak{d}, N(\mathfrak{t}), \mathfrak{k})+\mathbb{d} \sigma(\mathfrak{d}, \mathfrak{t}, N(\mathfrak{k}))=\mathbb{d} \sigma_{N}(\mathfrak{d}, \mathfrak{t}, \mathfrak{k}), \quad \forall \mathfrak{d}, \mathfrak{t}, \mathfrak{k} \in \Gamma(\mathfrak{D} E),
$$

where $\sigma_{N} \in \Gamma\left(\operatorname{Hom}\left(\wedge^{2} \mathfrak{D} E, E\right)_{\mathfrak{J} E}\right)$ is defined by

$$
\sigma_{N}(\mathfrak{d}, \mathfrak{t})=\sigma(N(\mathfrak{d}), \mathfrak{t}) .
$$

Proof. Consider the integrability condition (12). In fact, there are two equations since $\Gamma(\mathfrak{o l}(E))$ has two components $\Gamma(\mathfrak{D} E)$ and $\Gamma(\mathfrak{J} E)$. First let $e_{1}=\mu, e_{2}=\nu$ be elements in $\Gamma(\mathfrak{J} E)$, we have $\mathcal{J}(\mu)=\pi^{\sharp}(\mu)-N^{\star}(\mu), \mathcal{J}(\nu)=\pi^{\sharp}(\nu)-N^{\star}(\nu)$ and $\llbracket \mu, \nu \rrbracket=0$. Therefore, we obtain

$$
\begin{aligned}
& \llbracket \pi^{\sharp}(\mu)-N^{\star}(\mu), \pi^{\sharp}(\nu)-N^{\star}(\nu) \rrbracket-\mathcal{J}\left(\llbracket \pi^{\sharp}(\mu)-N^{\star}(\mu), \nu \rrbracket+\llbracket \mu, \pi^{\sharp}(\nu)-N^{\star}(\nu) \rrbracket\right) \\
= & {\left[\pi^{\sharp}(\mu), \pi^{\sharp}(\nu)\right]_{\mathfrak{D}}-\pi^{\sharp}\left(\mathfrak{L}_{\pi^{\sharp}(\mu)} \nu-i_{\pi^{\sharp}(\nu)} \mathbb{d} \mu\right) } \\
& +N^{\star}\left(\mathfrak{L}_{\pi^{\sharp}(\mu)} \nu-i_{\pi^{\sharp}(\nu)} \mathbb{d} \mu\right)-\mathfrak{L}_{\pi^{\sharp}(\mu)} N^{\star}(\nu)+i_{\pi^{\sharp}(\nu)} \mathbb{d} N^{\star}(\mu) \\
= & 0 .
\end{aligned}
$$

Thus we get conditions (17) and (19).

Then let $e_{1}=\mathfrak{d} \in \Gamma(\mathfrak{D} E)$ and $e_{2}=\mu \in \Gamma(\mathfrak{J} E)$, we have $\mathcal{J}\left(e_{1}\right)=N(\mathfrak{d})+\sigma_{\natural}(\mathfrak{d})$ and $\mathcal{J}\left(e_{2}\right)=$ $\pi^{\sharp}(\mu)-N^{\star}(\mu)$. Therefore, we obtain

$$
\begin{aligned}
& \left.\llbracket N(\mathfrak{d})+\sigma_{\natural}(\mathfrak{d}), \pi^{\sharp}(\mu)-N^{\star}(\mu) \rrbracket-\llbracket \mathfrak{d}, \mu \rrbracket-\mathcal{J}\left(\llbracket N(\mathfrak{d})+\sigma_{\natural}(\mathfrak{d})\right), \mu \rrbracket+\llbracket \mathfrak{d}, \pi^{\sharp}(\mu)-N^{\star}(\mu) \rrbracket\right) \\
= & {\left[N(\mathfrak{d}), \pi^{\sharp}(\mu)\right]_{\mathfrak{D}}-N\left[\mathfrak{d}, \pi^{\sharp}(\mu)\right]_{\mathfrak{D}}-\pi^{\sharp}\left(\mathfrak{L}_{N(\mathfrak{d})} \mu-\mathfrak{L}_{\mathfrak{d}} N^{\star}(\mu)\right) } \\
& +N^{\star}\left(\mathfrak{L}_{N(\mathfrak{d})} \mu-\mathfrak{L}_{\mathfrak{d}} N^{\star}(\mu)\right)-\mathfrak{L}_{N(\mathfrak{d})} N^{\star}(\mu)-i_{\pi^{\sharp}(\mu)} \mathbb{d} \sigma_{\natural}(\mathfrak{d})-\mathfrak{L}_{\mathfrak{d}} \mu-\sigma_{\natural}\left[\mathfrak{d}, \pi^{\sharp}(\mu)\right]_{\mathfrak{D}} \\
= & 0 .
\end{aligned}
$$


Thus we have

$$
\begin{aligned}
{\left[N(\mathfrak{d}), \pi^{\sharp}(\mu)\right]_{\mathfrak{D}} } & =N\left[\mathfrak{d}, \pi^{\sharp}(\mu)\right]_{\mathfrak{D}}+\pi^{\sharp}\left(\mathfrak{L}_{N(\mathfrak{d})} \mu-\mathfrak{L}_{\mathfrak{d}} N^{\star}(\mu)\right), \\
N^{\star}\left(\mathfrak{L}_{N(\mathfrak{d})} \mu-\mathfrak{L}_{\mathfrak{d}} N^{\star}(\mu)\right) & =\mathfrak{L}_{N(\mathfrak{d})} N^{\star}(\mu)+i_{\pi^{\sharp}(\mu)} \mathbb{d} \sigma_{\natural}(\mathfrak{d})+\mathfrak{L}_{\mathfrak{d}} \mu+\sigma_{\natural}\left[\mathfrak{d}, \pi^{\sharp}(\mu)\right]_{\mathfrak{D}} .
\end{aligned}
$$

We claim that (22) is equivalent to (19). In fact, applying (22) to $\nu \in \Gamma(\mathfrak{J} E)$ and (19) to $\mathfrak{d} \in \Gamma(\mathfrak{D} E)$, we get the same equality.

Next let $e_{1}=\mathfrak{d}$ and $e_{2}=\mathfrak{t}$ be elements in $\Gamma(\mathfrak{D} E)$, we have $\mathcal{J}\left(e_{1}\right)=N(\mathfrak{d})+\sigma_{\natural}(\mathfrak{d})$ and $\mathcal{J}\left(e_{2}\right)=N(\mathfrak{t})+\sigma_{\mathfrak{\natural}}(\mathfrak{t})$. Therefore, we have

$$
\begin{aligned}
& \llbracket N(\mathfrak{d})+\sigma_{\mathfrak{\natural}}(\mathfrak{d}), N(\mathfrak{t})+\sigma_{\mathfrak{\natural}}(\mathfrak{t}) \rrbracket-[\mathfrak{d}, \mathfrak{t}]_{\mathfrak{D}}-\mathcal{J}\left(\llbracket N(\mathfrak{d})+\sigma_{\mathfrak{\natural}}(\mathfrak{d}), \mathfrak{t} \rrbracket+\llbracket \mathfrak{d}, N(\mathfrak{t})+\sigma_{\mathfrak{\natural}}(\mathfrak{t}) \rrbracket\right) \\
= & {[N(\mathfrak{d}), N(\mathfrak{t})]_{\mathfrak{D}}-[\mathfrak{d}, \mathfrak{t}]_{\mathfrak{D}}-N\left([N(\mathfrak{d}), \mathfrak{t}]_{\mathfrak{D}}+[\mathfrak{d}, N(\mathfrak{t})]_{\mathfrak{D}}\right)-\pi^{\sharp}\left(\mathfrak{L}_{\mathfrak{d}} \sigma_{\mathfrak{\natural}}(\mathfrak{t})-i_{\mathfrak{t}} \mathfrak{d} \sigma_{\mathfrak{\natural}}(\mathfrak{d})\right) } \\
& +\mathfrak{L}_{N(\mathfrak{d})} \sigma_{\mathfrak{\natural}}(\mathfrak{t})-i_{N(\mathfrak{t})} \mathbb{d} \sigma_{\mathfrak{\natural}}(\mathfrak{d})-\sigma_{\mathfrak{\natural}}\left([N(\mathfrak{d}), \mathfrak{t}]_{\mathfrak{D}}+[\mathfrak{d}, N(\mathfrak{t})]_{\mathfrak{D}}\right)+N^{\star}\left(\mathfrak{L}_{\mathfrak{d}} \sigma_{\mathfrak{\natural}}(\mathfrak{t})-i_{\mathfrak{t}} \mathbb{d} \sigma_{\mathfrak{\natural}}(\mathfrak{d})\right) \\
= & 0 .
\end{aligned}
$$

Thus we have

$$
\begin{aligned}
{[N(\mathfrak{d}), N(\mathfrak{t})]_{\mathfrak{D}}-[\mathfrak{d}, \mathfrak{t}]_{\mathfrak{D}}-N\left([N(\mathfrak{d}), \mathfrak{t}]_{\mathfrak{D}}+[\mathfrak{d}, N(\mathfrak{t})]_{\mathfrak{D}}\right) } & =\pi^{\sharp}\left(\mathfrak{L}_{\mathfrak{d}} \sigma_{\mathfrak{\natural}}(\mathfrak{t})-i_{\mathfrak{t}} \mathbb{d} \sigma_{\natural}(\mathfrak{d})\right), \\
\sigma_{\mathfrak{\natural}}\left([N(\mathfrak{d}), \mathfrak{t}]_{\mathfrak{D}}+[\mathfrak{d}, N(\mathfrak{t})]_{\mathfrak{D}}\right)-\mathfrak{L}_{N(\mathfrak{d})} \sigma_{\natural}(\mathfrak{t})+i_{N(\mathfrak{t})} \mathbb{d} \sigma_{\natural}(\mathfrak{d}) & =N^{\star}\left(\mathfrak{L}_{\mathfrak{d}} \sigma_{\mathfrak{\natural}}(\mathfrak{t})-i_{\mathfrak{t}} \mathbb{d} \sigma_{\mathfrak{\natural}}(\mathfrak{d})\right) .
\end{aligned}
$$

We claim that (23) and (24) are equivalent. In fact, applying (23) and (24) to $t \in \Gamma(\mathfrak{D E})$ and $\mu \in \Gamma(\mathfrak{J} E)$ respectively, we get the same equality

$$
\begin{array}{r}
\left\langle[N(\mathfrak{d}), N(\mathfrak{t})]_{\mathfrak{D}}-[\mathfrak{d}, \mathfrak{t}]_{\mathfrak{D}}-N\left([N(\mathfrak{d}), \mathfrak{t}]_{\mathfrak{D}}+[\mathfrak{d}, N(\mathfrak{t})]_{\mathfrak{D}}\right), \mu\right\rangle_{E}=\mathfrak{d}\left\langle\pi^{\sharp} \sigma_{\sharp}(\mathfrak{t}), \mu\right\rangle_{E} \\
+\left\langle\sigma_{\sharp}(\mathfrak{t}),\left[\mathfrak{d}, \pi^{\sharp} \mu\right]_{\mathfrak{D}}\right\rangle_{E}+\mathfrak{t}\left\langle\sigma_{\sharp}(\mathfrak{d}), \pi^{\sharp}(\mu)\right\rangle_{E}-\pi^{\sharp}(\mu)\left\langle\sigma_{\sharp}(\mathfrak{d}), \mathfrak{t}\right\rangle_{E}-\left\langle\sigma_{\sharp}(\mathfrak{d}),\left[\mathfrak{t}, \pi^{\sharp}(\mu)\right]_{\mathfrak{D}}\right\rangle_{E} .
\end{array}
$$

By the equality $\pi^{\sharp} \circ \sigma_{\natural}+N^{2}=-$ id and (24), we have

$$
[N(\mathfrak{d}), N(\mathfrak{t})]_{\mathfrak{D}}+N^{2}[\mathfrak{d}, \mathfrak{t}]_{\mathfrak{D}}-N\left([N(\mathfrak{d}), \mathfrak{t}]_{\mathfrak{D}}+[\mathfrak{d}, N(\mathfrak{t})]_{\mathfrak{D}}\right)=\pi^{\sharp}\left(\mathfrak{L}_{\mathfrak{d}} \sigma_{\mathfrak{\natural}}(\mathfrak{t})-i_{\mathfrak{t}} \mathbb{d} \sigma_{\mathfrak{\natural}}(\mathfrak{d})-\sigma_{\mathfrak{\natural}}[\mathfrak{d}, \mathfrak{t}]_{\mathfrak{D}}\right),
$$

which implies that $T(N)(\mathfrak{d}, \mathfrak{t})=\pi^{\sharp}\left(i_{\mathfrak{d} \wedge} \mathfrak{t} d \sigma\right)$. Thus (24) is equivalent to (20).

At last, we consider condition (25). Acting on an arbitrary $\mathfrak{k} \in \Gamma(\mathfrak{D} E)$, we have

$$
\begin{aligned}
& N(\mathfrak{d})\left\langle\sigma_{\mathfrak{\natural}}(\mathfrak{t}), \mathfrak{k}\right\rangle_{E}-\left\langle\sigma_{\mathfrak{\natural}}(\mathfrak{t}),[N(\mathfrak{d}), \mathfrak{k}]_{\mathfrak{D}}\right\rangle_{E}+\left\langle\sigma_{\mathfrak{\natural}}(\mathfrak{k}),[N(\mathfrak{d}), \mathfrak{t}]_{\mathfrak{D}}\right\rangle_{E}-N(\mathfrak{t})\left\langle\sigma_{\mathfrak{\natural}}(\mathfrak{d}), \mathfrak{k}\right\rangle_{E} \\
& +\mathfrak{k}\left\langle\sigma_{\mathfrak{\natural}}(\mathfrak{d}), \mathfrak{t}\right\rangle_{E}+\left\langle\sigma_{\mathfrak{\natural}}(\mathfrak{d}),[N(\mathfrak{t}), \mathfrak{k}]_{\mathfrak{D}}\right\rangle_{E}+\left\langle\sigma_{\mathfrak{\natural}}(\mathfrak{k}),[\mathfrak{d}, N(\mathfrak{t})]_{\mathfrak{D}}\right\rangle_{E}+\mathfrak{d}\left\langle\sigma_{\mathfrak{\natural}}(\mathfrak{t}), N(\mathfrak{k})\right\rangle_{E} \\
& -\left\langle\sigma_{\mathfrak{\natural}}(\mathfrak{t}),[\mathfrak{d}, N(\mathfrak{k})]_{\mathfrak{D}}\right\rangle_{E}-\mathfrak{t}\left\langle\sigma_{\mathfrak{\natural}}(\mathfrak{d}), N(\mathfrak{k})\right\rangle_{E}+N(\mathfrak{k})\left\langle\sigma_{\mathfrak{\natural}}(\mathfrak{d}), \mathfrak{t}\right\rangle_{E}+\left\langle\sigma_{\mathfrak{\natural}}(\mathfrak{d}),[\mathfrak{t}, N(\mathfrak{k})]_{\mathfrak{D}}\right\rangle_{E} \\
= & \mathbb{d} \sigma(N(\mathfrak{d}), \mathfrak{t}, \mathfrak{k})+\mathfrak{t} \sigma(N(\mathfrak{d}), \mathfrak{k})-\mathfrak{k} \sigma(N(\mathfrak{d}), \mathfrak{t})+\sigma\left([\mathfrak{t}, \mathfrak{k}]_{\mathfrak{D}}, N(\mathfrak{d})\right) \\
& +\mathbb{d} \sigma(\mathfrak{d}, N(\mathfrak{t}), \mathfrak{k})-\mathfrak{d} \sigma(N(\mathfrak{t}), \mathfrak{k})-\sigma\left([\mathfrak{d}, \mathfrak{k}]_{\mathfrak{D}}, N(\mathfrak{t})\right) \\
& +\mathbb{d} \sigma(\mathfrak{d}, \mathfrak{t}, N(\mathfrak{k}))+\sigma\left([\mathfrak{d}, \mathfrak{t}]_{\mathfrak{D}}, N(\mathfrak{k})\right) \\
= & 0 .
\end{aligned}
$$

Note that the following equality holds:

$$
\sigma(\mathfrak{d}, N(\mathfrak{t}))=-\left\langle\sigma_{\mathfrak{\natural}}(N(\mathfrak{t})), \mathfrak{d}\right\rangle_{E}=-\left\langle N^{\star}\left(\sigma_{\mathfrak{\natural}}(\mathfrak{t})\right), \mathfrak{d}\right\rangle_{E}=-\left\langle\sigma_{\mathfrak{\natural}}(\mathfrak{t}), N(\mathfrak{d})\right\rangle_{E}=\sigma(N(\mathfrak{d}), \mathfrak{t}) .
$$

Therefore, we have

$$
\left(i_{N} \mathbb{d} \sigma\right)(\mathfrak{d}, \mathfrak{t}, \mathfrak{k})=\mathbb{d} \sigma_{N}(\mathfrak{d}, \mathfrak{t}, \mathfrak{k}),
$$

which implies that (25) is equivalent to (21). 
Remark 5.5. Let $\mathcal{J}=\left(\begin{array}{cc}N & \pi^{\sharp} \\ \sigma_{\natural} & -N^{\star}\end{array}\right)$ be a generalized complex structure on the omni-Lie algebroid $\mathfrak{o l}(E)$. Then $\pi$ satisfies (17). On one hand, in [4], the authors showed that such $\pi$ will give rise to a Lie bracket $[\cdot, \cdot]_{E}$ on $\Gamma(E)$ via

$$
[u, v]_{E}=\pi^{\sharp}(\mathbb{d} u)(v), \quad \forall u, v \in \Gamma(E) .
$$

On the other hand, by Theorem 4.6, the generalized complex structure $\mathcal{J}$ will also induce a Lie algebroid structure on $E$ by (13). By the equality

$$
\pi^{\sharp}=\rho \circ \mathcal{J} \circ \rho^{\star},
$$

these two Lie algebroid structures on $E$ are the same.

Remark 5.6. Recall that any $b \in \Gamma\left(\operatorname{Hom}\left(\wedge^{2} \mathfrak{D} E, E\right)_{\mathfrak{J} E}\right)$ defines a transformation $e^{b}: \mathfrak{o l}(E) \rightarrow$ ol $(E)$ defined by

$$
e^{b}\left(\begin{array}{c}
\mathfrak{d} \\
\mu
\end{array}\right)=\left(\begin{array}{cc}
\text { id } & 0 \\
b_{\natural} & \text { id }
\end{array}\right)\left(\begin{array}{l}
\mathfrak{d} \\
\mu
\end{array}\right)=\left(\begin{array}{c}
\mathfrak{d} \\
\mu+i_{\mathfrak{d}} b
\end{array}\right) .
$$

Thus, $e^{b}$ is an automorphism of the omni-Lie algebroid ol $(E)$ if and only if $\mathbb{d} b=0$. In this case, $e^{b}$ is called a $B$-field transformation. Genuinely, an automorphism of the omni-Lie algebroid ol $(E)$ is just the composition of an automorphism of the vector bundle $E$ and a B-field transformation. In fact $B$-field transformations map generalized complex structures on ol $(E)$ into new generalized complex structures as follows:

$$
\mathcal{J}^{b}=\left(\begin{array}{cc}
\text { id } & 0 \\
b_{\natural} & \text { id }
\end{array}\right) \circ \mathcal{J} \circ\left(\begin{array}{cc}
\text { id } & 0 \\
-b_{\natural} & \text { id }
\end{array}\right) .
$$

Example 5.7. Let $D: E \rightarrow E$ be a bundle map satisfying $D^{2}=-$ id. Define $R_{D}: \mathfrak{D} E \rightarrow \mathfrak{D} E$ by $R_{D}(\mathfrak{d})=\mathfrak{d} \circ D$ and $\hat{D}: \mathfrak{J} E \rightarrow \mathfrak{J} E$ by $\hat{D}(\mathbb{d} u)=\mathbb{d}(D u)$ for $u \in \Gamma(E)$. Then

$$
\mathcal{J}=\left(\begin{array}{cc}
R_{D} & 0 \\
0 & -\hat{D}
\end{array}\right)
$$

is a generalized complex structure on ol $(E)$. In fact, since

$$
\left\langle R_{D}^{\star}(\mathbb{d} u), \mathfrak{d}\right\rangle_{E}=\langle\mathbb{d} u, \mathfrak{d} \circ D\rangle_{E}=\mathfrak{d}(D(u))=\langle\hat{D}(\mathbb{d} u), \mathfrak{o}\rangle_{E},
$$

we have $R_{D}^{\star}=\hat{D}$. It is direct to check that the Nijenhuis tensor $T\left(R_{D}\right)$ vanishes and the condition $D^{2}=-$ id ensures that $R_{D}^{2}=-$ id.

Let $\pi \in \Gamma\left(\operatorname{Hom}\left(\wedge^{2} \mathfrak{J} E, E\right){ }_{\mathfrak{D} E}\right)$ and suppose that the induced map $\pi^{\sharp}: \mathfrak{J} E \rightarrow \mathfrak{D} E$ is an isomorphism of vector bundles. Then the rank of $E$ is 1 or equals to the dimension of $M$. We denote by $\left(\pi^{\sharp}\right)^{-1}$ the inverse of $\pi^{\sharp}$ and by $\pi^{-1}$ the corresponding element in $\Gamma\left(\operatorname{Hom}\left(\wedge^{2} \mathfrak{D} E, E\right)_{\mathfrak{J} E}\right)$.

Lemma 5.8. With the above notations, the following two statements are equivalent:

(i) $\pi \in \Gamma\left(\operatorname{Hom}\left(\wedge^{2} \mathfrak{J} E, E\right)_{\mathfrak{D} E}\right)$ satisfies (17);

(ii) $\pi^{-1}$ is closed, i.e. $\mathbb{d} \pi^{-1}=0$.

Proof. The conclusion follows from the following equality:

$$
\left\langle\pi^{\sharp}\left([\mu, \nu]_{\pi}\right)-\left[\pi^{\sharp}(\mu), \pi^{\sharp}(\nu)\right]_{\mathfrak{D}}, \gamma\right\rangle_{E}=-\mathbb{d} \pi^{-1}\left(\pi^{\sharp}(\mu), \pi^{\sharp}(\nu), \pi^{\sharp}(\gamma)\right), \quad \forall \mu, \nu, \gamma \in \Gamma(\mathfrak{J} E),
$$


which can be obtained by straightforward computations.

Let $\left(E,[\cdot, \cdot]_{E}, a\right)$ be a Lie algebroid. Define $\pi^{\sharp}: \mathfrak{J} E \rightarrow \mathfrak{D} E$ by

$$
\pi^{\sharp}(\mathbb{d} u)(\cdot)=[u, \cdot]_{E}, \quad \forall u \in \Gamma(E) .
$$

Then $\pi^{\sharp}$ satisfies (17). Furthermore, $\left(\mathfrak{J} E,[\cdot, \cdot]_{\pi}, \mathfrak{j} \circ \pi^{\sharp}\right)$ is a Lie algebroid, where the bracket $[\cdot, \cdot]_{\pi}$ is given by (18). By Theorem 5.4 and Lemma 5.8, we have

Corollary 5.9. Let $\left(E,[\cdot, \cdot]_{E}, a\right)$ be a Lie algebroid such that the induced map $\pi^{\sharp}: \mathfrak{J} E \rightarrow \mathfrak{D} E$ is an isomorphism. Then

$$
\mathcal{J}=\left(\begin{array}{cc}
0 & \pi^{\sharp} \\
-\left(\pi^{\sharp}\right)^{-1} & 0
\end{array}\right),
$$

is a generalized complex structure on ol $(E)$.

Example 5.10. Let $\left(T M,[\cdot, \cdot]_{T M}\right.$, id) be the tangent Lie algebroid. Define $\pi^{\sharp}: \mathfrak{J}(T M) \rightarrow \mathfrak{D}(T M)$ by

$$
\pi^{\sharp}(\mathbb{d} u)=[u, \cdot]_{T M} .
$$

Then $\pi^{\sharp}$ is an isomorphism. See [4, Corollary 3.9] for details. Then

$$
\mathcal{J}=\left(\begin{array}{cc}
0 & \pi^{\sharp} \\
-\left(\pi^{\sharp}\right)^{-1} & 0
\end{array}\right),
$$

is a generalized complex structure on the omni-Lie algebroid of $(T M)$.

Example 5.11. Let $(M, \omega)$ be a symplectic manifold and $\left(T^{*} M,[\cdot, \cdot]_{\omega^{-1}},\left(\omega^{\sharp}\right)^{-1}\right)$ the associated natural Lie algebroid. Define $\pi^{\sharp}: \mathfrak{J}\left(T^{*} M\right) \rightarrow \mathfrak{D}\left(T^{*} M\right)$ by

$$
\pi^{\sharp}(\mathbb{d} u)=[u, \cdot]_{\omega^{-1}},
$$

which is an isomorphism (see [4, Corollary 3.10]). Then

$$
\mathcal{J}=\left(\begin{array}{cc}
0 & \pi^{\sharp} \\
-\left(\pi^{\sharp}\right)^{-1} & 0
\end{array}\right),
$$

is a generalized complex structure on the omni-Lie algebroid $\mathfrak{o l}\left(T^{*} M\right)$.

At the end of this section, we introduce the notion of an algebroid-Nijenhuis structure, which can give rise to generalized complex structures on the omni-Lie algebroid orl $(E)$.

Definition 5.12. Let $\left(E,[\cdot, \cdot]_{E}, a\right)$ be a Lie algebroid, $N: \mathfrak{D} E \longrightarrow \mathfrak{D} E$ a Nijenhuis operator on the Lie algebroid $\left(\mathfrak{D} E,[\cdot, \cdot]_{\mathfrak{D}}, \mathfrak{\mathfrak { j }}\right)$ satisfying $N^{\star}(\mathfrak{J} E) \subset \mathfrak{J} E$ and $\pi: \mathfrak{J} E \longrightarrow \mathfrak{D} E$ given by (28). $N$ and $\pi$ are said to be compatible if

$$
N \circ \pi^{\sharp}=\pi^{\sharp} \circ N^{\star}, \quad \text { and } \quad C(\pi, N)=0,
$$

where

$$
C(\pi, N)(\mu, \nu) \triangleq[\mu, \nu]_{\pi_{N}}-\left(\left[N^{\star}(\mu), \nu\right]_{\pi}+\left[\mu, N^{\star}(\nu)\right]_{\pi}-N^{\star}[\mu, \nu]_{\pi}\right), \quad \forall \mu, \nu \in \Gamma(\mathfrak{J} E) .
$$

Here $\pi_{N} \in \Gamma\left(\operatorname{Hom}\left(\wedge^{2} \mathfrak{J} E, E\right)_{\mathfrak{D} E}\right)$ is given by

$$
\pi_{N}(\mu, \nu)=\left\langle\nu, N \pi^{\sharp}(\mu)\right\rangle_{E}, \quad \forall \mu, \nu \in \Gamma(\mathfrak{J} E) .
$$

If $N$ and $\pi$ are compatible, we call the pair $(\pi, N)$ an algebroid-Nijenhuis structure on the Lie algebroid $\left(E,[\cdot, \cdot]_{E}, a\right)$. 
The following lemma is straightforward, we omit the proof.

Lemma 5.13. Let $\left(E,[\cdot, \cdot]_{E}, a\right)$ be a Lie algebroid, $\pi$ given by (28) and $N: \mathfrak{D} E \longrightarrow \mathfrak{D} E$ a Nijenhuis structure. Then $(\pi, N)$ is an algebroid-Nijenhuis structure on the Lie algebroid $\left(E,[\cdot, \cdot]_{E}, a\right)$ if and only if $N \circ \pi^{\sharp}=\pi^{\sharp} \circ N^{\star}$ and

$$
N^{\star}[\mu, \nu]_{\pi}=\mathfrak{L}_{\pi(\mu)} N^{\star}(\nu)-\mathfrak{L}_{\pi(\nu)} N^{\star}(\mu)-\mathbb{d} \pi\left(N^{\star}(\mu), \nu\right) .
$$

By Theorem 5.4 and Lemma 5.13 , we have

Theorem 5.14. Let $\left(E,[\cdot, \cdot]_{E}, a\right)$ be a Lie algebroid, $\pi$ given by (28) and $N: \mathfrak{D} E \longrightarrow \mathfrak{D} E a$ Nijenhuis structure. Then the following statements are equivalent:

(a) $(\pi, N)$ is an algebroid-Nijenhuis structure and $N^{2}=-\mathrm{id}$;

(b) $\mathcal{J}=\left(\begin{array}{cc}N & \pi^{\sharp} \\ 0 & -N^{\star}\end{array}\right)$ is a generalized complex structure on the omni-Lie algebroid $\mathfrak{o l}(E)$.

Remark 5.15. An interesting special case is that where $E=L$ is a line bundle. Then $(\pi, N)$ becomes a Jacobi-Nijenhuis structure on $M$. Jacobi-Nijenhuis structures are studied by Luca Vitagliano and the third author in [25]. In this case, $\pi$ defines a Jacobi bi-derivation $\{\cdot, \cdot\}$ of $L$ (i.e. a skew-symmetric bracket which is a first order differential operator, hence a derivation, in each argument). Moreover, this bi-derivation is compatible with $N$ in the sense that $\pi^{\sharp} N^{\star}=N \circ \pi^{\sharp}$ and $C(\pi, N)=0$. It defines a new Jacobi bi-derivation $\{\cdot, \cdot\}_{N}$. Furthermore, $\left(\{\cdot, \cdot\},\{\cdot, \cdot\}_{N}\right)$ is a Jacobi bi-Hamiltonian structure, i.e. $\{\cdot, \cdot\},\{\cdot, \cdot\}_{N}$ and $\{\cdot, \cdot\}+\{\cdot, \cdot\}_{N}$ are all Jacobi brackets.

\section{Generalized complex structures on omni-Lie algebras}

In this section, we consider the case that $E$ reduces to a vector space $V$. Then we have

$$
\mathfrak{D} V=\operatorname{gl}(V), \quad \mathfrak{J} V=V .
$$

Furthermore, the pairing (4) reduces to

$$
\langle A, u\rangle_{V}=A u, \quad \forall A \in \operatorname{gl}(V), u \in V .
$$

Any $u \in V$ is a linear map from $\mathrm{gl}(V)$ to $V$,

$$
u(A)=\langle A, u\rangle_{V}=A u .
$$

Therefore, an omni-Lie algebroid reduces to an omni-Lie algebra, which is introduced by Weinstein in [26] to study the linearization of the standard Courant algebroid.

Definition 6.1. An omni-Lie algebra associated to $V$ is a triple $\left(\operatorname{gl}(V) \oplus V, \llbracket \cdot, \cdot \rrbracket,(\cdot, \cdot)_{V}\right)$, where $(\cdot, \cdot)_{V}$ is a nondegenerate symmetric pairing given by

$$
(A+u, B+v)_{V}=\frac{1}{2}(A v+B u), \quad \forall A, B \in \operatorname{gl}(V), u, v \in V,
$$

and $\llbracket \cdot, \cdot \rrbracket$ is a bracket operation given by

$$
\llbracket A+u, B+v \rrbracket=[A, B]+A v .
$$

We will simply denote an omni-Lie algebra associated to a vector space $V$ by orl $V)$. 
Lemma 6.2. For any vector space $V$, we have

$$
\begin{aligned}
\operatorname{Hom}\left(\wedge^{2} \mathrm{gl}(V), V\right)_{V} & =0, \\
\operatorname{Hom}\left(\wedge^{2} V, V\right)_{\operatorname{gl}(V)} & =\operatorname{Hom}\left(\wedge^{2} V, V\right) .
\end{aligned}
$$

Proof. In fact, for any $\phi \in \operatorname{Hom}\left(\wedge^{2} \operatorname{gl}(V), V\right)_{V}$ and $A, B \in \operatorname{gl}(V)$, by (15), we have

$$
\phi(A \wedge B)=B \circ \phi\left(A \wedge \mathrm{id}_{V}\right)=-B \circ A \circ \phi\left(\mathrm{id}_{V} \wedge \mathrm{id}_{V}\right)=0 .
$$

Therefore, $\phi=0$, which implies that $\operatorname{Hom}\left(\wedge^{2} \operatorname{gl}(V), V\right)_{V}=0$. The second equality is obvious.

Proposition 6.3. Any generalized almost complex structure $\mathcal{J}: \operatorname{gl}(V) \oplus V \longrightarrow \operatorname{gl}(V) \oplus V$ on the omni-Lie algebra orl $(V)$ is of the following form

$$
\left(\begin{array}{cc}
-R_{D} & \pi^{\sharp} \\
0 & D
\end{array}\right)
$$

where $\pi \in \operatorname{Hom}\left(\wedge^{2} V, V\right), D \in \operatorname{gl}(V)$ satisfying $D^{2}=-\mathrm{id}_{V}$ and $\pi(D u, v)=\pi(u, D v)$, and $R_{D}$ : $\mathrm{gl}(V) \longrightarrow \mathrm{gl}(V)$ is the right multiplication, i.e. $R_{D}(A)=A \circ D$.

Proof. By Proposition 5.2 and Lemma 6.2 we can assume that a generalized almost complex structure is of the form $\left(\begin{array}{cc}N & \pi^{\sharp} \\ 0 & -N^{\star}\end{array}\right)$, where $N: \operatorname{gl}(V) \longrightarrow \operatorname{gl}(V)$ satisfies $N^{\star} \in \operatorname{gl}(V)$ and $N^{2}=-\mathrm{id}_{\mathrm{gl}(V)}$, and $\pi \in \operatorname{Hom}\left(\wedge^{2} V, V\right)$. Let $D=-N^{\star}$, then we have

$$
(D v)(A)=-N^{\star}(v)(A)=-v(N(A))=-N(A) v .
$$

On the other hand, we have $(D v)(A)=A D v$, which implies that $N(A)=-R_{D}(A)$. It is obvious that $N^{2}=-\mathrm{id}_{\mathrm{gl}(V)}$ is equivalent to that $D^{2}=-\mathrm{id}_{V}$. The proof is finished.

Theorem 6.4. A generalized almost complex structure $\mathcal{J}: \operatorname{gl}(V) \oplus V \longrightarrow \operatorname{gl}(V) \oplus V$ on the omni-Lie algebra oll $(V)$ given by (33) is a generalized complex structure if and only if

(i) $\pi$ defines a Lie algebra structure $[\cdot, \cdot]_{\pi}$ on $V$;

(ii) $D^{2}=-\mathrm{id}_{V}$ and $D[u, v]_{\pi}=[u, D v]_{\pi}$ for $u, v \in V$.

Thus, a generalized complex structure on the omni-Lie algebra ol $(V)$ gives rise to a complex Lie algebra structure on $V$.

Proof. By Theorem [5.4, we have

$$
[u, v]_{\pi}=\pi^{\sharp}(u)(v)=\pi(u, v) .
$$

Condition (17) implies that $[\cdot, \cdot]_{\pi}$ gives a Lie algebra structure on $V$. Condition (19) implies that $D[u, v]_{\pi}=[u, D v]_{\pi}$. The other conditions are valid.

The conditions $D^{2}=-\mathrm{id}_{V}$ and $D[u, v]_{\pi}=[u, D v]_{\pi}$ say by definition that $D$ is a complex Lie algebra structure on $\left(V,[\cdot, \cdot]_{\pi}\right)$. This finishes the proof.

\section{References}

[1] J. Barton and M. Stiénon, Generalized complex submanifolds, Pacific J. Math. 236 (2008), no. $1,23-44$. 
[2] D. Baraglia, Conformal Courant algebroids and orientifold T-duality, Int. J. Geom. Methods Mod. Phys. 10 (2013), no. 2, 1250084, 35 pp.

[3] H. Bursztyn, G. Cavalcanti and M. Gualtieri, Reduction of Courant algebroids and generalized complex structures, Adv. Math. 211 (2007), no. 2, 726-765.

[4] Z. Chen and Z. J. Liu, Omni-Lie algebroids, J. Geom. Phys. 60 (2010), no. 5, 799-808.

[5] Z. Chen and Z. J. Liu and Y. H. Sheng, E-Courant algebroids, Int. Math. Res. Notices (2010), no. $22,4334-4376$.

[6] M. Crainic, Generalized complex structures and Lie brackets, Bull. Braz. Math. Soc. (N.S.) 42 (2011), no. 4, 559-578.

[7] J. Grabowski and G. Marmo, The graded Jacobi algebras and (co)homology, J. Phys. A: Math. Gen. 36 (2003), 161-81.

[8] M. Gualtieri, Generalized complex geometry, Ann. of Math. (2) 174 (2011), no. 1, 75-123.

[9] N. J. Hitchin, Generalized Calabi-Yau manifolds, Q. J. Math. 54 (2003), no. 3, 281-308.

[10] D. Iglesias-Ponte and A. Wade, Contact manifold and generalized complex structures, $J$. Geom. Phys. 53 (2005), 249-258.

[11] M. Jotz Lean, $N$-manifolds of degree 2 and metric double vector bundles, arXiv:1504.00880,

[12] A. Kirillov, Local Lie algebras, Russian Math. Surveys 31 (1976), 55-76.

[13] H. Lang, Y. Sheng and X. Xu, Nonabelian omni-Lie algebras, Banach Center Publications 110 (2016), 167-176.

[14] D. Li-Bland, AV-Courant algebroids and generalized CR structures, Canad. J. Math. 63 (2011), no. 4, 938-960.

[15] D. Li-Bland, $\mathcal{L} \mathcal{A}$-Courant algebroids and their applications, thesis, University of Toronto, 2012, arXiv:1204.2796v1.

[16] D. Li-Bland and E. Meinrenken, Courant algebroids and Poisson geometry, Int. Math. Res. Not. IMRN, 11 (2009), 2106-2145.

[17] Z. J. Liu, A. Weinstein and P. Xu, Manin triples for Lie bialgebroids, J. Diff. Geom. 45 (1997), 547-574.

[18] K. Mackenzie, General theories of Lie groupoids and Lie algebroids, Cambridge University Press, 2005.

[19] K. Mackenzie, Ehresmann doubles and Drindel'd doubles for Lie algebroids and Lie bialgebroids, J. Reine Angew. Math. 658 (2011), 193-245.

[20] D. Roytenberg, Courant algebroids, derived brackets and even symplectic supermanifolds, PhD thesis, UC Berkeley, 1999, arXiv:math.DG/9910078.

[21] Y. Sheng, On deformations of Lie algebroids, Results. Math. 62 (2012), 103-120.

[22] M. Stiénon and P. Xu, Poisson quasi-Nijenhuis manifolds, Comm. Math. Phys. 270 (2007), no. $3,709-725$. 
[23] M. Stiénon and P. Xu, Reduction of generalized complex structures, J. Geom. Phys. 58 (2008), no. 1, 105-121.

[24] L. Vitagliano and A. Wade, Generalized contact bundles, C. R. Math. Acad. Sci. Paris 354 (2016), 313-317.

[25] L. Vitagliano and A. Wade, Holomorphic Jacobi manifolds, arXiv:1609.07737.

[26] A. Weinstein, Omni-Lie algebras, Microlocal analysis of the Schrödinger equation and related topics (Japanese) (Kyoto, 1999), Sūrikaisekikenkyūsho Kōkyūroku 1176(2000), 95-102. 